\title{
Breast cancer in women using digoxin: tumor characteristics and relapse risk
}

Robert J Biggar ${ }^{1,2^{*}}$, Elisabeth W Andersen ${ }^{1}$, Niels Kroman ${ }^{3}$, Jan Wohlfahrt ${ }^{1}$ and Mads Melbye ${ }^{1}$

\begin{abstract}
Introduction: Digoxin use is associated with increased incidence of breast and uterus cancers. We postulated that digoxin use might affect tumor characteristics and increase relapse risk in women with breast cancer.

Methods: Incident breast cancer cases in Danish women ( $n=49,312 ; 1995$ to 2008) were identified. Analyses were conducted in women 20 to 74 years old. Relapse hazard ratios (HR) were compared in women using and not using digoxin, adjusting for age, calendar period, protocol, tumor size, nodal involvement, histology grade, estrogen-receptor (ER) status, and anti-estrogen therapy in Cox regression models.

Results: At diagnosis, tumors in digoxin users were more likely ER+ (85.4\% vs. $78.6 \%: P=0.002)$ and have grade 1 ductal histology (37.2\% vs. $25.7 ; P=0.004)$, compared to non-users. 45 relapses occurred in women already using digoxin at breast cancer diagnosis (1,487 person-years; 24 relapses occurred in women later starting digoxin (384 person-years). Overall relapse HR in digoxin users was 1.13 (95\% confidence interval: $0.88,1.46)$ compared to non-users. Relapse risk in digoxin users was significantly increased in the first year $(2.19 ; 1.26,3.78)$ but not thereafter $(0.99 ; 0.74,1.32)(P=0.02$ for difference in HRs). First-year relapse hazard was high in digoxin-using women with ER+ tumors $(2.51 ; 1.39,4.55)$ but not ER- tumors $(0.72 ; 0.10,5.27)$. Recurrence hazard was not significantly changed among digoxin-using women also using tamoxifen.
\end{abstract}

Conclusions: Breast cancers arising in digoxin-using women had better prognostic features. After adjustment for markers, overall breast cancer relapse risk in digoxin users was not increased significantly, although recurrence hazards for ER+ tumors were higher in the first year following diagnosis.

\section{Introduction}

Digoxin is a phyto-estrogen widely used in the care of patients with heart disease, where its effect is mediated by the $\mathrm{Na}^{+} \mathrm{K}^{+}$-ATPase ion pump [1]. However, it also binds to the estrogen receptor (ER) and appears to be capable of causing gynecomastia [2]. Women currently using digoxin are at $30 \%$ to $50 \%$ increased risk of developing incident breast [3,4] and uterus [5] cancer compared to non-users, likely through estrogen-mimicking activity [2]. In Denmark, exogenous estrogen use also has been reported to increase relapse risk in women with a history of breast cancer and, in practice, use is contraindicated for this group [6,7]. We therefore examined if clinically relevant tumor characteristics [8] or relapse risk might differ in women who developed breast

\footnotetext{
* Correspondence: rjbiggar@gmail.com

'Department of Epidemiology Research, Statens Serum Institut, Artillerivej 5, Copenhagen, 2300, Denmark

Full list of author information is available at the end of the article
}

cancer while currently using digoxin, adjusting for age, calendar year and protocol.

A priori, we predicted that any effects on recurrence would be most apparent in women with ER-positive rather than ER-negative cancers, given that digoxin likely exerts its effect via attachment to the ER [2]. In the past two decades, anti-estrogen therapies have become increasingly used in women with ER-positive tumors and might affect an impact from digoxin. The two major classes of antiestrogen drugs act by different mechanisms. Tamoxifen and drugs of this class (hereafter referred to as tamoxifen) interfere with ER binding [9] and might diminish any deleterious effects of digoxin respect to recurrence. In contrast, aromatase inhibitors (AIs), now commonly used in postmenopausal women, reduce systemic estrogen levels but would not be expected to affect digoxin-related excess risks [10]. Therefore, we also predicted that any digoxinrelated risk of relapse, would be higher in women on AIs than those on tamoxifen [2].

\section{() Biomed Central}




\section{Materials and methods}

Cases were identified through data in the Danish Breast Cancer Cooperative Group [11], which has centrally recorded information on women with breast cancer in Denmark since 1978. Heart disease requiring digoxin use is a serious co-morbid condition affecting overall prognosis. Because we could not confidently determine the precise cause of death, we chose first relapse as our outcome. Among women entering treatment protocols (mostly those with stage 1 and 2), relapse was defined as the first appearance of new tumor (local/regional recurrence, contralateral breast cancer, or metastatic disease). Relapse information was recorded only for women enrolled in protocols, and therefore patients not in protocols were excluded. The current study included women diagnosed from 1 January 1995 and followed patients from diagnosis to first relapse, death, loss to follow-up, or to 31 December 2008, whichever came first. Patient linkage in registry data was undertaken by authorized national registries and the investigators received only anonymous and untraceable grouped data for analysis. Approvals for this study were obtained in advance from the Danish Ethical Review Board and the Danish Data Protection Agency.

Registry information included diagnosis date, age at diagnosis, tumor size, number of positive lymph nodes, histology grading, ER status, and anti-estrogen therapy. We obtained information on digoxin use from the Danish Register of Medicinal Products Statistics [12], a nationwide centralized registry, which has recorded individual prescriptions for pharmaceutical drugs since 1 January 1995. Digoxin is the only digitalis preparation in the Danish formulary (CO1AA05 in the Anatomical Therapeutic Chemical (ATC) classification, available from the World Health Organization Collaborating Centre for Drug Statistics Methodology, Norwegian Institute of Public Health, PO BOX 4404 Nydalen, NO-0304, Oslo, Norway). Postmenopausal use of estrogen-containing preparations has declined since 1995 and is uncommon in women more than 70 years old [13], which is the group most likely to be using digoxin [2].

Evaluation included women using digoxin at breast cancer diagnosis and those who began using digoxin after breast cancer was diagnosed. Biomarkers affecting prognosis (histology, tumor size and nodal involvement) were evaluated among those already using digoxin at breast cancer diagnosis, adjusted for age, calendar year and protocol. Hazard ratios (HRs) for recurrence were assessed in those continuing or starting digoxin after diagnosis. Those starting digoxin after diagnosis were considered unexposed until they started by including digoxin use as a time-dependent variable. Women were considered digoxin users only for the time of current use, considered to be six months after their most recent prescription. Thus, in time-dependent analyses, a woman was reclassified as a non-user six months after a prescription, unless she filled a new prescription. We have previously used this measure, finding that increased breast cancer incidence was only seen during the time of current digoxin use [4].

Protocol criteria usually exclude women with advanced cancers or complicated co-morbid conditions, since such patients may need individualized care outside standard guidelines. Additionally, older or ill women were more likely to refuse protocol therapy. Due to missing relapse information, patients not in protocols could not be used. We examined demographic and tumor characteristics at breast cancer diagnosis by age. Selecting age $\leq 74$ years provided the maximum inclusion of cases with protocolaccessible data (Figure 1). As was done in previous studies $[4,5]$, we examined HRs in women currently using angina drugs but not digoxin, as a comparison for breast cancer risk in women with heart disease. Angina-drug exposure was treated as a time-dependent variable in which digoxin users were excluded (in other words, angina drug users compared to neither angina nor digoxin users), as previously defined [4]. The angina drug group included both nitrites and nicorandil (ATC codes: CO1DA02, CO1DA08, CO1DA014, CO1DX16). We previously reported that angina drug use did not affect the incidence of breast [4] or uterus cancer [5]. Angina drugs were started at a slightly younger age than digoxin (median age at first drug use in all women since 1995, 70 and 78 years, respectively [5]).

Exposure to tamoxifen and/or AIs was identified by patient allocation to arms of protocols that included these drugs. Details of drug type, exposure duration and dose were not available by individual. When used, anti-estrogen treatments were typically started within 4 to 8 weeks of diagnosis. The AIs are now commonly used in postmenopausal patients with breast cancer [10], the age group that most commonly uses digoxin. However, AI use did not become widespread until 2005 [10]. Therefore, follow-up time in AI users was limited. Furthermore, many potential users were still enrolled in blinded protocols (individual use unknown) and could not be enrolled in this sub-analysis, further limiting power. In the earlier years of this study, most women using anti-estrogen therapies were taking tamoxifen, regardless of their age. In analysis of the impact of treatment, patients were grouped by those exposed to tamoxifen only, AIs only, and those who took neither (54\% of all patients). Patients who received both tamoxifen and AIs at any time during their treatment course (22\%), and those still in blinded protocols (3\%) were excluded from analysis of the impact of anti-estrogen therapy on the HR for relapse in digoxin users. 


\section{All patients}

\section{9,312}

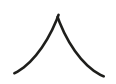

Age at onset

$$
\leq 74 \text { y/o }
$$

$39,987(81.9 \%)$

$$
\geq 75 \mathrm{y} / \mathrm{o}
$$

$9.325(18.9 \%)$

\begin{tabular}{|c|c|c|c|}
\hline Yes & $\mathrm{No}^{1}$ & Yes & $\mathrm{No}^{1,2}$ \\
\hline $4(86.2 \%)$ & $5,533(13.8 \%)$ & $2,318(24.9 \%)$ & $7,007(75.1 \%)$ \\
\hline
\end{tabular}

\section{Protocol enrolled}

\section{On digoxin at enrollment}<smiles>CCCC</smiles>

Yes

$369(1.1 \%) \quad 34,085$

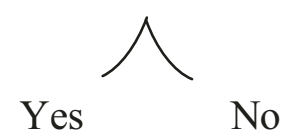

$113(2.1 \%)$
No

5,420

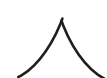

Yes

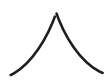

Yes

No

Figure 1 Women over 20 years old with breast cancer in Denmark, 1995 to 2008 . ${ }^{1}$ Excluded from analysis of relapse because relapse data were not available; ${ }^{2}$ includes 26 patients (none on digoxin) who were assigned to protocol but who accrued no time on protocol treatment;

${ }^{3}$ main analysis file.

Relapse risk was compared in women using digoxin to those not using digoxin by calculating the HR with $95 \%$ CIs using Cox regression models [14]. Age profiles differed between digoxin users and non-users. Therefore, the HR for recurrence was adjusted for age at breast cancer diagnosis (20 to 39 years old, and in 5-year age groups thereafter). Six protocols were used during the study time period, each with different criteria (for example, low- or high-risk cancers). We therefore adjusted for protocol. Since therapy improvements likely reduced HRs for relapse during the study period, we also adjusted for calendar year period (1995 to 1999, 2000 to 2004, and 2005 to 2008). Finally we adjusted for tumor size, histology grade, number of positive nodes, and ER status at time of diagnosis (unless being evaluated in analysis).

In Figure 2, log-HRs with 95\% CIs were estimated from a locally weighted scatterplot smoothed (LOESS)- curve of the scaled Schoenfeld residuals [15] for digoxin exposure for each relapse time. In primary analysis, subjects with missing data were excluded, but in sensitivity analyses, patients with missing data were included with imputation of missing information [16]. The imputation models were ordinal logistic for tumor size, positive nodes and histology grading and included all the variables available at baseline as well as log person-years (py) and relapse status as explanatory variables. Missing information within each variable was assumed to be random, and 10 imputations were included in the model. The distribution of the imputed values was found to be similar to those in the original data.

\section{Results}

We identified 49,312 women with incident breast cancer in the study period, of whom 1,454 (2.9\%) used digoxin 


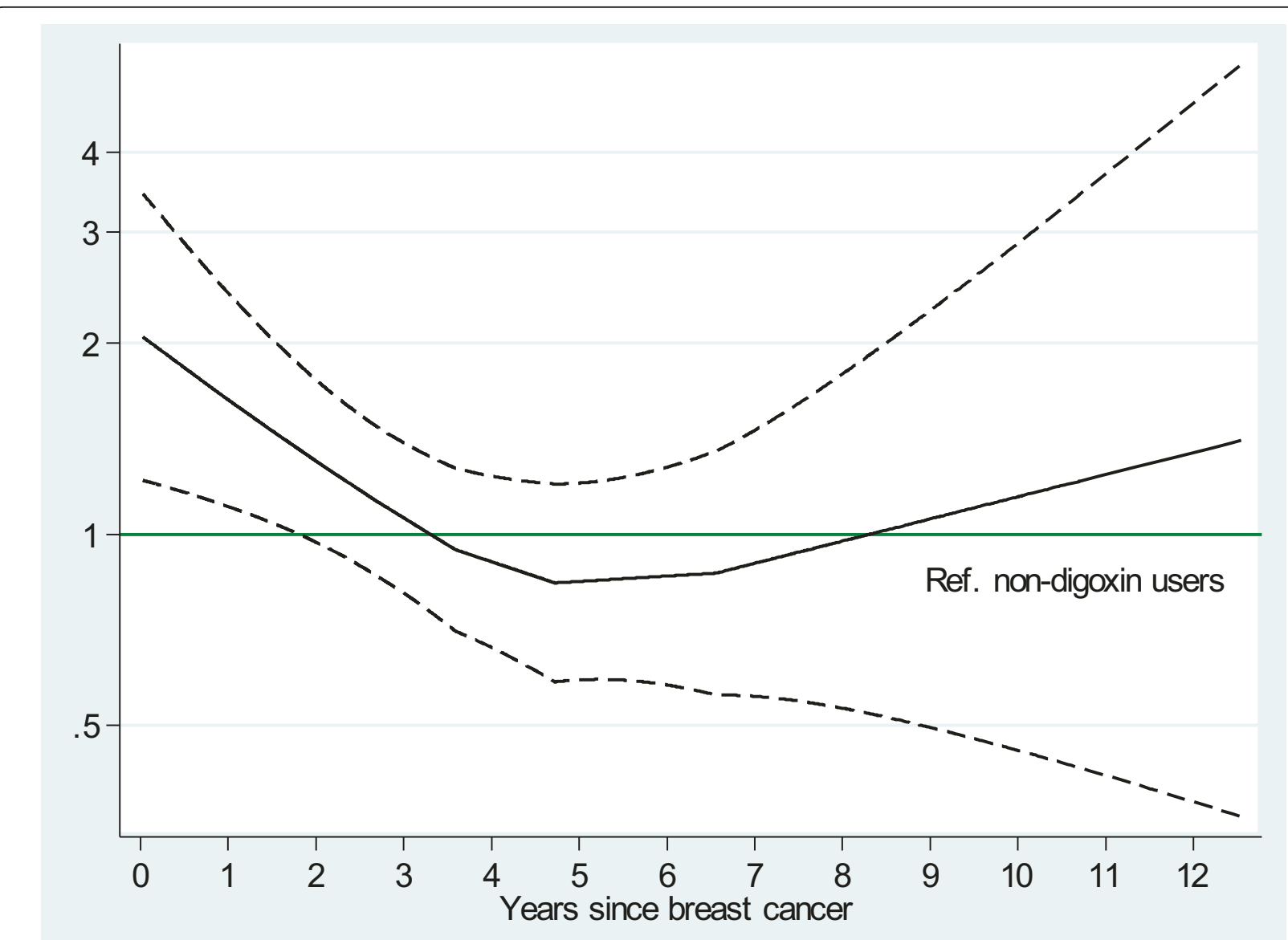

Figure 2 Estimated relapse hazard ratio $(95 \% \mathrm{Cl})$ for digoxin-exposed compared to unexposed (referent) women with breast cancer, adjusting for current age group and stratifying by protocol, protocol year, histological grade, node status, tumor size, and estrogen receptor status.

at breast cancer diagnosis. However, 9,325 patients were $\geq 75$ years old, among whom the majority $(75.1 \%)$ were not enrolled in protocol studies (Figure 1) and hence lacked relapse information. In patients $>75$ years old, digoxin use was more frequent in non-participants $(11.5 \%)$ than in protocol enrollees $(7.0 \%)$. Among patients $\leq 74$ years old, $86.2 \%$ participated in protocol studies and tumor characteristics were $97.2 \%$ complete (Table 1 ). In women $\leq 74$ years old, digoxin use was also more common in non-participants (2.1\%) than in participants (1.1\%).

Among 34,454 women $\leq 74$ years old who contributed time in protocol-treated breast cancer studies in Denmark since 1995, 369 were on digoxin at breast cancer diagnosis (Figure 1). Patients on digoxin at diagnosis had 1,487 py of follow-up (average: 4.0 years; 45 relapses). Additional patients later started digoxin, contributing 384 py of follow-up (24 relapses). In total, during 1,872 py of follow-up while using digoxin, there were 69 relapses. In 34,085 patients not using digoxin at breast cancer diagnosis, there were 148,418 py of follow-up without digoxin use (average: 4.4 years; 4,647 relapses).
Among patients with information available, tumors arising in women who were using digoxin were more likely to be ER-positive $(P=0.002)$ and have grade I ductal histology $(P=0.004)$, and to have less nodal involvement $(P=$ 0.14 ) than among patients not on digoxin. In 33,496 patients $(97.2 \%)$ with complete covariate information, the overall HR for relapse in women currently using digoxin was not statistically different than in those not using digoxin (HR 1.13, 95\% CI 0.88, 1.46), after adjustment for age group, calendar-year of cancer diagnosis, protocol, histology grade, positive nodes, tumor size, and ER status (Table 2). However, in the first year, the HR for relapse was $2.19(1.26,3.78)$, comparing digoxin-users to nonusers (Table 3). After the first year, the HR for relapse in patients on digoxin was $0.99(0.74,1.32)$, which was significantly lower than for women using digoxin during the first year $(P=0.02)$. When HRs were permitted to differ between first and subsequent years, digoxin significantly increased the HR for relapse $(P=0.04)$.

Among women with ER-positive breast cancers, 13 relapses occurred in digoxin users during the first year 
Table 1 Patients, relapse and follow-up in women with breast cancer by use of digoxin

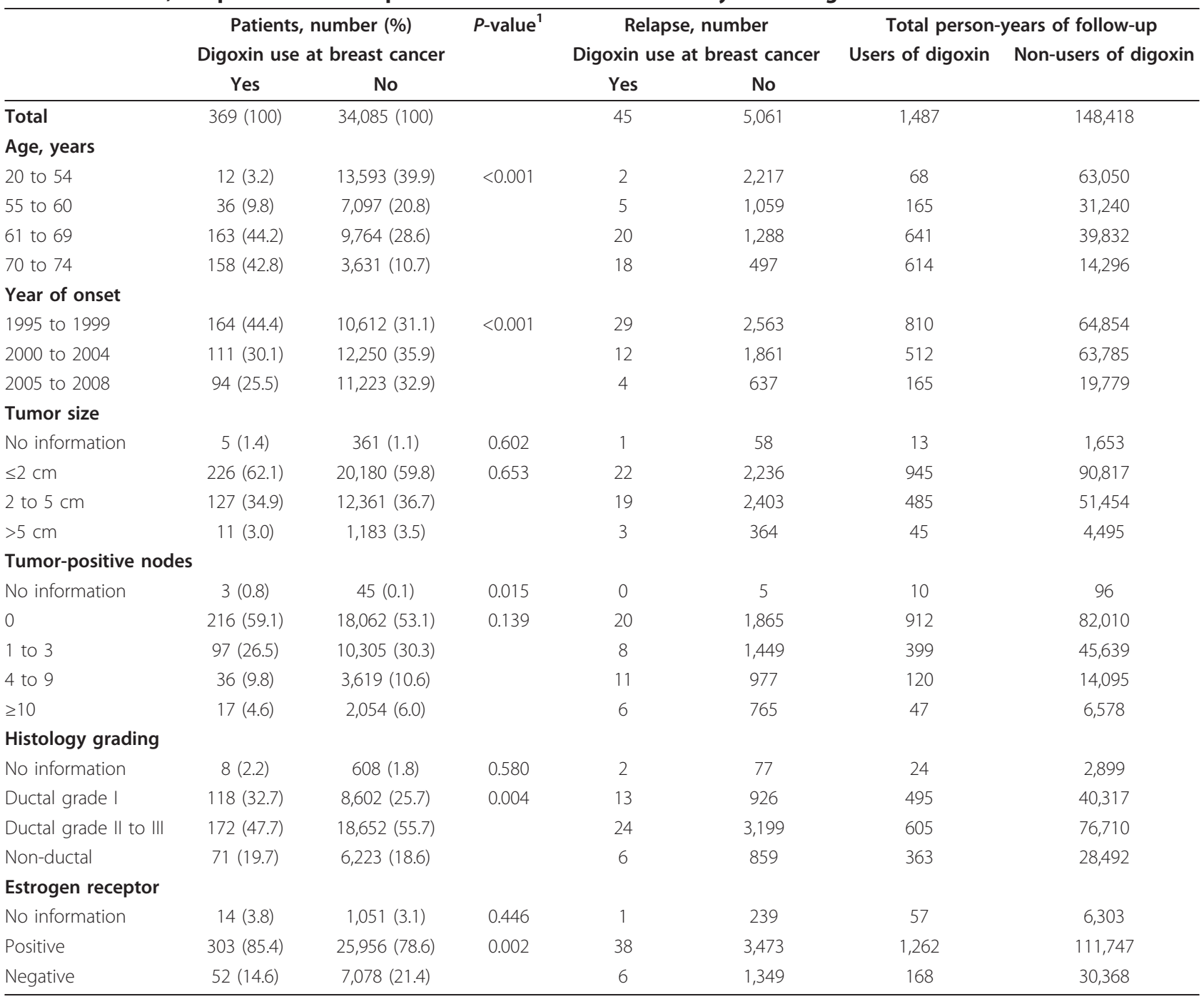

${ }^{1} \mathrm{Chi}^{2}$ tests for comparison of group with no information to those with information, and heterogeneity among groups within strata with known data.

after diagnosis (HR 2.51, 95\% CI 1.39, 4.55) (Table 3). In women presenting with ER-negative tumors, only one relapse occurred in digoxin users during the first year. The resulting HR for digoxin use, $0.72(0.10,5.27)$, was low but statistically unstable, and the difference in HR between women with ER-positive and ER-negative tumors was not statistically significant $(P=0.24)$. After the first year, the HR for relapse with digoxin use was similar in women with ER-positive (HR 0.98, 95\% CI $0.71,1.36)$ and ER-negative (HR 0.97, 95\% CI 0.45, 2.09) breast cancers $(P=0.98)$.

To determine if tamoxifen or AI use affected the HR for digoxin-related relapse, we examined anti-estrogen therapy assigned to each ER-positive patient (Table 2). In the small subset of digoxin-using patients who also got a single type of anti-estrogen therapy, relapse occurred in twenty-six patients using tamoxifen only and four patients using AIs only. Among currentdigoxin users, the overall HR for relapse in women only exposed to tamoxifen was 1.04 (95\% CI 0.68, 1.60), whereas in women only using AIs, the HR was 1.87 $(0.46,7.56)$. This difference in relapse HRs was not statistically significant $(P=0.43)$.

As a sensitivity analysis of the effect of digoxin on the HR for relapse, we re-analyzed outcomes on all 34,454 protocol-enrolled women, imputing missing data in the $2.8 \%$ women with incomplete confounder information and using fully adjusted models. The results were similar to those in the main analysis. In the first year after diagnosis, the HR for relapse was 2.22 (95\% CI 1.30, 3.77). For those exposed in periods after the first year following diagnosis, the HR was $0.98(0.74,1.30)$ among current digoxin-users. Thus, missing data did not affect our findings. 
Table 2 Overall relapse hazard ratios for 33,496 women $<74$ years old with breast cancer and using digoxin

\begin{tabular}{|c|c|c|c|c|c|}
\hline & Digoxin-exposed & Person-years & Relapse, number & Hazard ratio $(95 \% \mathrm{Cl})$ & $P$-value ${ }^{1}$ \\
\hline \multirow[t]{2}{*}{$\overline{\text { All }}$} & Yes & 1,872 & 69 & $1.13(0.88,1.46)$ & 0.04 \\
\hline & No & 148,034 & 5,037 & 1.0 & \\
\hline \multicolumn{6}{|c|}{ Estrogen receptor status } \\
\hline \multirow[t]{2}{*}{ Positive } & Yes & 1,524 & 55 & $1.15(0.87,1.52)$ & 0.03 \\
\hline & No & 111,485 & 3,456 & 1.0 & \\
\hline \multirow[t]{2}{*}{ Negative } & Yes & 280 & 10 & $0.93(0.45,1.90)$ & 0.94 \\
\hline & No & 30,256 & 1,345 & 1.0 & \\
\hline \multirow[t]{2}{*}{ Unknown } & Yes & 67 & 4 & $1.67(0.49,5.69)$ & 0.53 \\
\hline & No & 6,293 & 236 & 1.0 & \\
\hline \multicolumn{6}{|c|}{ Digoxin exposure with anti-estrogen treatment ${ }^{2}$} \\
\hline \multirow[t]{2}{*}{ Tamoxifen } & Yes & 353 & 26 & $1.04(0.68,1.60)$ & 0.36 \\
\hline & No & 23,742 & 1,431 & 1.0 & \\
\hline \multirow[t]{2}{*}{ Aromatase inhibitors } & Yes & 79 & 4 & $1.87(0.46,7.56)$ & 0.68 \\
\hline & No & 2,467 & 87 & 1.0 & \\
\hline \multirow[t]{2}{*}{ Neither } & Yes & 1,038 & 33 & $1.10(0.75,1.61)$ & 0.49 \\
\hline & No & 81,704 & 2,815 & 1.0 & \\
\hline
\end{tabular}

Risk compares patients (complete information only) using digoxin to those not using digoxin (referent) overall and by first and subsequent years after breast cancer diagnosis, by estrogen receptor status and allocated anti-estrogen treatment regimen. ${ }^{1}$ The $P$ - value evaluates the overall digoxin effect on the hazard ratios for relapse, permitting different digoxin effects in the first year and later years of follow-up. ${ }^{2}$ Not presented are risks in subjects who switched between tamoxifen and aromatase inhibitors (that is, they were exposed to both at some time in their treatment) and those still in blinded protocols (exposure unknown).

Table 3 Hazard ratios for breast cancer relapse by time period of digoxin exposure

\begin{tabular}{|c|c|c|c|c|c|c|c|}
\hline & \multirow[b]{2}{*}{$\begin{array}{l}\text { Digoxin- } \\
\text { exposed }\end{array}$} & \multicolumn{2}{|c|}{ First year } & \multirow[b]{2}{*}{$\begin{array}{l}\text { Hazard ratio } \\
(95 \% \mathrm{Cl})\end{array}$} & \multicolumn{2}{|c|}{ Subsequent years } & \multirow[b]{2}{*}{$\begin{array}{l}\text { Hazard ratio }(95 \% \\
\text { Cl) }\end{array}$} \\
\hline & & $\begin{array}{l}\text { Person- } \\
\text { years }\end{array}$ & $\begin{array}{l}\text { Relapse, } \\
\text { number }\end{array}$ & & $\begin{array}{l}\text { Person- } \\
\text { years }\end{array}$ & $\begin{array}{l}\text { Relapse, } \\
\text { number }\end{array}$ & \\
\hline \multirow[t]{2}{*}{ All } & Yes & 350 & 15 & $\begin{array}{l}2.19(1.26 \\
3.78)\end{array}$ & 1,521 & 54 & $0.99(0.74,1.32)$ \\
\hline & No & 30891 & 699 & 1,0 & 11,7143 & 4,338 & 1.0 \\
\hline \multicolumn{8}{|c|}{ Estrogen receptor status } \\
\hline \multirow[t]{2}{*}{ Positive } & Yes & 285 & 13 & $\begin{array}{l}2.51(1.39 \\
4.55)\end{array}$ & 1,239 & 42 & $0.98(0.71,1.36)$ \\
\hline & No & 23,524 & 405 & 1.0 & 87,961 & 3,051 & 1.0 \\
\hline \multirow[t]{2}{*}{ Negative } & Yes & 51 & 1 & $\begin{array}{l}0.72(0.10 \\
5.27)\end{array}$ & 229 & 9 & $0.97(0.45,2.09)$ \\
\hline & No & 6,404 & 262 & 1.0 & 23,852 & 1,083 & 1 \\
\hline \multirow[t]{2}{*}{ Unknown } & Yes & 15 & 1 & $\begin{array}{l}4.29(0.43 \\
42.4)\end{array}$ & 53 & 3 & $1.27(0.29,5.58)$ \\
\hline & No & 963 & 32 & 1.0 & 5,330 & 204 & 1.0 \\
\hline \multicolumn{8}{|c|}{ Digoxin exposure with anti-estrogen treatment ${ }^{1}$} \\
\hline \multirow[t]{2}{*}{ Tamoxifen } & Yes & 71 & 6 & $\begin{array}{l}1.90(0.81 \\
4.67)\end{array}$ & 282 & 20 & $0.89(0.54,1.47)$ \\
\hline & No & 5,382 & 185 & 1.0 & 18,360 & 1,246 & 1.0 \\
\hline \multirow[t]{2}{*}{$\begin{array}{l}\text { Aromatase } \\
\text { inhibitors }\end{array}$} & Yes & 21 & 1 & $\begin{array}{l}2.39(0.26 \\
22.4)\end{array}$ & 58 & 3 & $1.63(0.28,9.43)$ \\
\hline & No & 1,037 & 12 & 1.0 & 1,430 & 75 & \\
\hline \multirow[t]{2}{*}{ Neither } & Yes & 193 & 8 & $\begin{array}{l}1.76(0.74 \\
4.21)\end{array}$ & 846 & 25 & $1.00(0.65,1.54)$ \\
\hline & No & 16,141 & 461 & 1.0 & 65,563 & 2.354 & 1.0 \\
\hline
\end{tabular}

Risk compares patients (complete information only) using digoxin to those not using digoxin (referent) overall and by first and subsequent years after breast cancer diagnosis, by estrogen receptor status and allocated anti-estrogen treatment regimen. ${ }^{1}$ Among tamoxifen and aromatase inhibitor users, hazard ratios for relapse in digoxin users for the first year and and at one year did not differ $(P=0.85$ and $P=0.52$, respectively). 
In a further sensitivity analysis, we also re-analyzed the dataset of protocol-enrolled women with complete confounder information using patients of all ages and applying the same adjustments. The patterns were again similar. Among 1,077 women currently using digoxin, 81 relapses occurred during 2,191 py of follow-up. After complete adjustment, the overall HR among women currently using digoxin was 1.15 (95\% CI 0.91, 1.46), compared to the HR for those using neither drug. In the first year, the HR for relapse was 2.40 (95\% CI 1.52, 3.77). For digoxin exposures more than one year after diagnosis, the HR for relapse was $0.95(0.72,1.26)$, without significant variation in the HR by years after diagnosis in this period. Thus, the age restriction did not affect the findings.

Finally, we analyzed the HR for relapse among women $\leq 74$ years old who were using angina drugs but not digoxin. After adjustment for all variables used in the digoxin analyses, the overall HR was 1.40 (95\% CI 1.11, 1.75). The HR for relapse in the first year was 1.40 $(0.71,2.74)$ and for following years, the HR was also $1.40(1.10,1.78)$.

\section{Discussion}

Women taking digoxin had an increased proportion of ER-positive low-grade tumors and less nodal involvement compared to those not taking digoxin at the time of diagnosis. While there was no statistically significant difference in overall risk of relapse for women taking vs. not taking digoxin for the entire follow-up period, recurrence risk was statistically significantly increased (HR 2.2) in the first year after cancer diagnosis in women taking digoxin. Although statistically different from the HR for relapse in digoxin users during subsequent years, the increased risk of recurrence in the first year could just be an unusual chance finding.

Thus, the recurrence risk patterns for digoxin use are similar to those reported for postmenopausal hormone replacement therapy, which is also associated with an increase in breast cancer incidence $[17,18]$. Specifically, women who develop breast cancer while on estrogens also have a higher proportion of ER-positive better-prognosis tumors [8] and those who continue or are placed on estrogens also have an increased recurrent risk [6,7]. Use of progestin-containing hormonal combinations has been proposed to increase risk of recurrence more than estrogen alone [19]. In this regard, the pharmacology of digoxin would resemble that of estrogen-alone use, assuming it acts as an estrogen-agonist. Long-term survival appears to be better in those who develop breast cancer while using estrogen [20-23], perhaps because the tumors have better prognosis characteristics, but we did not examine survival in digoxin-users because of concern about the impact of co-morbidity from cardiac conditions.
An objective of this study was to examine the effect of anti-estrogen therapy on the effects of digoxin. Use of tamoxifen reduces progression of ER-positive ductal carcinoma in situ $[9,24]$. If digoxin mediates its effect through the ER, then blocking the ER with tamoxifen might interfere with a digoxin effect. In contrast, AI acts by blocking estrogen production, leaving the ERs intact and digoxin without competition for attachment to the ER [2]. The modest changes in HR with tamoxifen or AI use are possibly consistent with mediation of a digoxin effect through the ER, but we acknowledge that these results were not statistically significant and require further evaluation. A further consideration is whether digoxin acts via interaction with the ER or via an interaction with the $\mathrm{Na}^{+} \mathrm{K}^{+}$-ATPase pump, the major target of digoxin activity [1]. The observation that changes in risk are specific to ER-positive tumors argues for a digoxin effect mediated via ER interaction.

The strengths of this study are its large size, populationrepresentativeness, and completeness. A high proportion of women $\leq 74$ years old were enrolled in protocols and the modest losses due to exclusion did not influence outcomes. High quality data were available about the patients, their tumors, the therapies they received, and recurrence outcomes. During this period, there was no awareness of the potential risks of digoxin use during this period, and therefore digoxin use would not be related to the cancer treatment regimen. Studies of anti-estrogen therapies were restricted to patients with ER-positive tumors who were randomly selected within the protocol assignment to receive them. They were therefore unbiased by the indication for anti-estrogen therapy. Being under observation for cardiac problems might accelerate the recognition of relapse. However, all women in their first year after entering therapy for breast cancer will be under close scrutiny for relapse, regardless of their cardiac condition, and a two-fold HR increase seems unlikely.

We also evaluated risk of relapse in women using vasodilating drugs but not digoxin, to be sure that women with cardiac conditions did not have exceptional risk of relapse for reasons other than digoxin use. To our surprise, women using vasodilators were at significantly increased risk of relapse in both the first year and subsequent years of follow-up. Vasodilators have no estrogen-mimicking properties, are not associated with gynecomastia, and do not increase the incidence of breast cancer [4]. Women with cardiac conditions might have received other drugs that induce gynecomastia, which we have speculated might increase breast cancer incidence [2]. We acknowledge that unknown confounders might have influenced the associations observed for both digoxin and angina drugs.

The association of relapse with digoxin and angina drugs potentially could suggest that risk might be attributable to 
heart disease, possibly mediated through obesity. In our study, measures of obesity were not available. Postmenopausal breast cancer incidence is high in obese women $[25,26]$. The cancers are especially likely to be ER-positive, and the route is thought to be related to higher levels of estrogen, which are also high in older obese women [27]. Older obese women are also at increased risk of cancer diagnosis in the contralateral breast $[27,28]$. Similarly, there is ample evidence that obese women have poorer survival rates following ER-positive breast cancer [29-32]). However, obesity is associated with increased mortality regardless of coincident breast cancer [33].

The effect of obesity on risk of recurrence is unclear, but in those studies reporting differences, the risk in normal-weight and obese women begins to diverge at only 3 to 4 years following treatment $[29,31,32]$. Whether these recurrences represent new cancers or true relapses is unknown. Obesity is not associated with increased risk of relapse in the first year after diagnosis, the period in which we observed an increased HR with digoxin use. Thus, we cannot attribute the two-fold increased HR for relapse in the first year to an interaction with obesity. However, we cannot exclude confounding from another unknown co-factor associated with heart disease.

\section{Conclusions}

While breast cancer incidence is increased in the women taking digoxin $[3,4]$, the breast cancers occurring in women taking digoxin had better prognostic features than in women not using digoxin. After adjustment for prognostic features, women who continued to use digoxin after breast cancer onset had a significantly higher risk of recurrence in the first year but not overall vs. those not using digoxin.

\section{Abbreviations}

Al: aromatase inhibitor; ATC: anatomical therapeutic chemicals; ER: estrogen receptor; HR: hazard ratio; LOESS: locally weighted scatterplot smoothing; py: person-years; $n$ : number of observations.

\section{Authors' contributions}

$\mathrm{RB}$ conceived of and organized the study and was primarily responsible for drafting the manuscript; EA analyzed the data; NK collected primary data and provided clinical input; JW obtained the dataset and guided statistical analysis; MM helped with its design and coordination. All authors assisted with drafting the manuscript and approved the final manuscript.

\section{Competing interests}

The authors declare that they have no competing interests.

\section{Acknowledgements}

Funding was provided by the Kraeftens Bekaempelse (Danish Cancer Society), Grant \#R40-A1857-11-S2. The Agency had no role in the study data, design, execution, conclusions or decision to publish.

\section{Author details}

'Department of Epidemiology Research, Statens Serum Institut, Artillerivej 5, Copenhagen, 2300, Denmark. ${ }^{2}$ Institute of Health and Biomedical Innovation, Queensland University of Technology, 60 Musk Avenue, Brisbane, 4059,
Australia. ${ }^{3}$ Danish Breast Cancer Cooperative Group and Department of Breast Surgery, Rigshospitalet, Blegdamsvej 9, Copenhagen, 2100, Denmark.

Received: 28 June 2012 Revised: 9 January 2013

Accepted: 8 February 2013 Published: 19 February 2013

1. McDonough AA, Velotta JB, Schwinger RH, Philipson KD, Farley RA: The cardiac sodium pump: structure and function. Basic Res Cardiol 2002, 97(Suppl 1):119-24.

2. Biggar RJ: Molecular pathways: digoxin use and estrogen-sensitive cancers-risks and possible therapeutic implications. Clin Cancer Res 2012, 18:2133-2137.

3. Ahern TP, Lash TL, Sørensen HT, Petersen L: Digoxin treatment is associated with an increased incidence of breast cancer: a populationbased case control study. Breast Cancer Res 2008, 10:R102.

4. Biggar RJ, Wohlfahrt J, Oudin A, Hjuler T, Mads Melbye: Digoxin use and the risk of breast cancer in women. J Clin Oncol 2011, 29:2165-2170.

5. Biggar RJ, Wohlfahrt J, Melbye M: Digitalis use and cancers of the corpus uteri, ovary and cervix. Int J Cancer 2012, 131:716-721.

6. Holmberg L, Anderson H, HABITS steering and data monitoring committees: HABITS (hormonal replacement therapy after breast canceris it safe?) A randomized comparison: trial stopped. Lancet 2004, 363:453-455.

7. Holmberg L, Iversen $\mathrm{OE}$, Rudenstam CM, Hammar M, Kumpulainen $E_{\text {, }}$ Jaskiewicz J, Jassem J, Dobaczewska D, Fjosne HE, Peralta O, Arriagada R, Holmqvist M, Maenpaa J, HABITS Study Group: Increased risk of recurrence after hormone replacement therapy in breast cancer survivors. $J \mathrm{NCl}$ 2008, 100:475-482

8. Colditz GA, Baer HJ, Tamimi RM: Breast Cancer. In Cancer Epidemiology and Prevention. Edited by: Schottenfeld D, Fraumeni JF Jr. New York: Oxford University Press, Inc; 2006:995-1012.

9. Rivera-Guevara C, Camacho J: Tamoxifen and its new derivatives in cancer research. Recent Pat Anticancer Drug Discov 2011, 6:237-245.

10. Chumsri S, Howes T, Bao T, Sabnis G, Brodie A: Aromatase, aromatase inhibitors, and breast cancer. J Steroid Biochem Mol Biol 2011, 125:13-22.

11. Moller $S$, Jensen MB, Ejlertsen $B$, Bjerre KD, Larsen M, Hansen HB, Christiansen P, Mouridsen HT, Danish Breast Cancer Cooperative Group: The clinical database and the treatment guidelines of the Danish Breast Cancer Cooperative Group (DBCG); its 30-years experience and future promise. Acta Oncol 2008, 47:506-524.

12. Gaist D, Sorensen HT, Hallas J: The Danish prescription registries. Dan Med Bull 1997, 44:445-448.

13. Lokkegaard E, Lidegaard O, Moller LN, Agger C, Andreasen AH, Jorgensen T: Hormone replacement therapy in Denmark, 1995-2004. Acta Obstet Gynecol Scand 2007, 86:1342-1351.

14. Cox DR: Regression models and life-tables (with discussion). J Royal Statistical Society, Series B 1972, 34: 187-220.

15. Grambsch PM, Therneau TM: Proportional hazards tests and diagnostics based on weighted residuals. Biometrika 1994, 81:515-526.

16. Little RJA, Rubin DB: Statistical Analysis with Missing Data. 2 edition. Hoboken, NJ: Wiley; 2002.

17. Collaborative Group on Hormonal Factors in Breast Cancer: Breast cancer and hormone replacement therapy: collaborative reanalysis of data from 51 epidemiological studies of 52,705 women with breast cancer and 108,411 women without breast cancer. Lancet 1997, 350:1047-1059.

18. Rossouw JE, Anderson GL, Prentice RL, LaCroix AZ, Kooperberg C, Stefanick ML, Jackson RD, Beresford SA, Howard BV, Johnson KC, Kotchen JM, Ockene J, Writing Group for the Women's Health Initiative Investigators: Risks and benefits of estrogen plus progestin in healthy postmenopausal women: principal results From the Women's Health Initiative randomized controlled trial. JAMA 2002, 288:321-333.

19. Chlebowski RT, Anderson GL: Progestins and recurrence in breast cancer survivors. J Natl Cancer Inst 2005, 97:471-472.

20. Chen W, Petitti D, Geiger A: Mortality following development of breast cancer while using oestrogen or oestrogen plus progestin: a computer record-linkage study. Br J Cancer 2005, 93:392-398.

21. Cheek J, Lacy J, Toth-Fejel S, Morris K, Calhoun K, Pommier RF: The impact of hormone replacement therapy on the detection and stage of breast cancer. Arch Surg 2002, 137:1015-1019. 
22. Bonnier P, Romain S, Giacalone PL, Laffargue F, Martin PM, Piana L: Clinical and biologic prognostic factors in breast cancer diagnosed during postmenopausal hormone replacement therapy. ᄀObstet Gynecol 1995, 85:11-17.

23. Newcomb PA, Egan KM, Trentham-Dietz A, Titus-Ernstoff L, Baron JA, Hampton JM, Stampfer MJ, Willett WC: Prediagnostic use of hormone therapy and mortality after breast cancer. Cancer Epidemiol Biomarkers Prev 2008, 17:864-871.

24. Alfred DC, Anderson SJ, Wickerham DL, Nagtegaal ID, Swain SM, Mamounas EP, Julian TB, Geyer CE Jr, Costantino JP, Land SR, Wolmark N: Adjuvant tamoxifen reduces subsequent breast cancer in women with estrogen receptor-positive ductal carcinoma in situ: a study based on NSABP Protocol B-24. J Clin Oncol 2012, 30:1268-1273.

25. Harvie M, Hooper L, Howell AH: Central obesity and breast cancer risk: a systematic review. Obes Rev 2003, 4:157-173.

26. Wolin KY, Carson K, Colditz GA: Obesity and cancer. Oncologist 2010, 15:556-565.

27. Dignam JJ, Wieand K, Johnson KA, Fisher B, Xu L, Mamounas EP: Obesity, tamoxifen use, and outcomes in women with estrogen receptor-positive early-stage breast cancer. J Natl Cancer Inst 2003, 95:1467-1476.

28. Majed B, Dozol A, Ribassin-Majed L, Senouci K, Asselain B: Increased risk of contralateral breast cancers among overweight and obese women: a time-dependent association. Breast Cancer Res Treat 2011, 126:729-738.

29. Sparano JA, Wang M, Zhao F, Stearns V, Martino S, Ligibel JA, Perez EA, Saphner T, Wolff AC, Sledge GW Jr, Wood WC, Fetting J, Davidson NE: Obesity at diagnosis is associated with inferior outcomes in hormone receptor-positive operable breast cancer. Cancer 2012, 118:5937-5946.

30. Protani $\mathrm{M}$, Coory $\mathrm{M}$, Martin $\mathrm{JH}$ : Effect of obesity on survival of women with breast cancer: systematic review and meta-analysis. Breast Cancer Res Treat 2010, 123:627-635.

31. Ewertz $M$, Jensen $M B$, Gunnarsdóttir KÁ, Højris I, Jakobsen EH, Nielsen $D$, Stenbygaard LE, Tange UB, Cold S: Effect of obesity on prognosis after early-stage breast cancer. J Clin Oncol 2011, 29:25-31.

32. de Azambuja E, McCaskill-Stevens W, Francis P, Quinaux E, Crown JP, Vicente M, Giuliani R, Nordenskjöld B, Gutiérez J, Andersson M, Vila MM, Jakesz R, Demol J, Dewar J, Santoro A, Lluch A, Olsen S, Gelber RD, Di Leo A, Piccart-Gebhart M: The effect of body mass index on overall and disease-free survival in node-positive breast cancer patients treated with docetaxel and doxorubicin-containing adjuvant chemotherapy: the experience of the BIG 02-98 trial. Late relapse typically assoc with ER+ disease. J Clin Oncol 1996, 14:2738-2746.

33. Schelbert KB: Comorbidities of obesity. Prim Care 2009, 36:271-285.

doi: $10.1186 / \mathrm{bcr} 3386$

Cite this article as: Biggar et al:: Breast cancer in women using digoxin: tumor characteristics and relapse risk. Breast Cancer Research 201315 R13.

\section{Submit your next manuscript to BioMed Central and take full advantage of:}

- Convenient online submission

- Thorough peer review

- No space constraints or color figure charges

- Immediate publication on acceptance

- Inclusion in PubMed, CAS, Scopus and Google Scholar

- Research which is freely available for redistribution 